Mechanical low-frequency filter via modes separation in 3D periodic structures

L. D'Alessandro, E. Belloni, R. Ardito, F. Braghin, and A. Corigliano

Citation: Appl. Phys. Lett. 111, 231902 (2017);

View online: https://doi.org/10.1063/1.4995554

View Table of Contents: http://aip.scitation.org/toc/apl/111/23

Published by the American Institute of Physics 


\title{
Mechanical low-frequency filter via modes separation in 3D periodic structures
}

\author{
L. D'Alessandro, ${ }^{1}$ E. Belloni, ${ }^{2}$ R. Ardito, ${ }^{1}$ F. Braghin, ${ }^{2}$ and A. Corigliano ${ }^{1, a)}$ \\ ${ }^{1}$ Department of Civil and Environmental Engineering, Politecnico di Milano, Piazza Leonardo da Vinci, \\ 32, 20133 Milano, Italy \\ ${ }^{2}$ Department of Mechanical Engineering, Politecnico di Milano, Via Giuseppe La Masa, 1, 20156 Milano, \\ Italy
}

(Received 12 July 2017; accepted 10 November 2017; published online 5 December 2017)

\begin{abstract}
This work presents a strategy to design three-dimensional elastic periodic structures endowed with complete bandgaps, the first of which is ultra-wide, where the top limits of the first two bandgaps are overstepped in terms of wave transmission in the finite structure. Thus, subsequent bandgaps are merged, approaching the behaviour of a three-dimensional low-pass mechanical filter. This result relies on a proper organization of the modal characteristics, and it is validated by performing numerical and analytical calculations over the unit cell. A prototype of the analysed layout, made of Nylon by means of additive manufacturing, is experimentally tested to assess the transmission spectrum of the finite structure, obtaining good agreement with numerical predictions. The presented strategy paves the way for the development of a class of periodic structures to be used in robust and reliable wave attenuation over a wide frequency band. Published by AIP Publishing.

https://doi.org/10.1063/1.4995554
\end{abstract}

Periodic structures find numerous applications in physics and engineering domains due to their peculiar properties in wave guiding and filtering. A major example in the electromagnetic field is represented by photonic crystals, ${ }^{1,2}$ while their counterparts for acoustic and elastic waves are the so-called phononic crystals. ${ }^{3,4}$ Focusing on elastic periodic structures, the frequency range of applications is wide: ${ }^{5}$ from extremely high frequencies, i.e., THz region for heat transmission, ${ }^{6}$ to few $\mathrm{Hz}$ in the seismic metamaterial domain, ${ }^{7}$ often taking the most from locally resonant mechanisms. ${ }^{8-12}$ Among others, the bandgap (i.e., the frequency range of prevented wave transmission) is one of the most investigated properties: a wide and complete bandgap is generally beneficial to guarantee robust wave attenuation around a certain frequency. ${ }^{4,13}$ In many cases, a complete bandgap is obtained by a periodic arrangement of two or more materials, ${ }^{4,14}$ but significant results can also be achieved for a single material, ${ }^{15-17}$ among which the one endowed with a very large complete bandgap that the authors show in a previous paper. ${ }^{18}$ In general, periodic structures endowed with bandgaps exhibit attenuation in the transmission spectrum of the finite structure in correspondence of the bandgap frequency range. ${ }^{4,14,15,18}$ Conversely, the design presented in this paper is such that the transmission spectrum of the finite structure is typical of a low-pass mechanical filter: the attenuation starts in correspondence of the bandgap bottom limit, and it proceeds beyond the bandgap top limits, merging the subsequent bandgaps. This behaviour is due to the design strategy adopted for the unit cell of the periodic structure, which is presented both analytically and numerically, that confines the mechanical energy of the structure in well separated frequency bands by exploiting the peculiar features of modal shapes. The elastic modes are organized such that all modes characterized by big modal

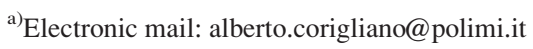

masses are confined in the first passband, while, from the second on, all the passbands are characterized by small modal masses, i.e., local modes. The bandgap merging is confirmed by both numerical calculations and experimental tests on a finite prototype. Additionally, a comparison with a prototype of a homogeneous solid cube of the same material, production process, and dimensions is carried out to highlight the differences between the proposed design and the bulk material.

The periodic structure analysed is shown in Fig. 1(a), while a cross section of the unit cell is presented in Fig. 1(b). The geometrical characteristics of the unit cell are [refer to Fig. 1(b)] $w_{1}=0.04 a, w_{2}=0.05 a, l=1.05(a-2 r)$, with $a$ being the unit cell characteristic dimension and $r=0.33 a$ the sphere radius. For the prototyping and experimental activity, the unit cell size is chosen as $a=0.05 \mathrm{~m}$, while the material is Nylon PA12 ${ }^{19}$ (Young's modulus $E=1.586 \mathrm{GPa}$, Poisson's ratio $\nu=0.4$, density $\rho=1000 \mathrm{~kg} / \mathrm{m}^{3}$, and sound velocity $v=\sqrt{E / \rho}=1259 \mathrm{~m} / \mathrm{s})$.

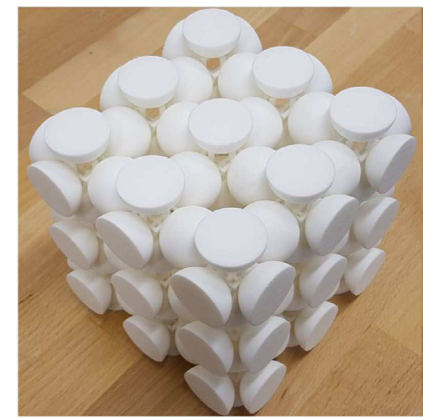

(a)

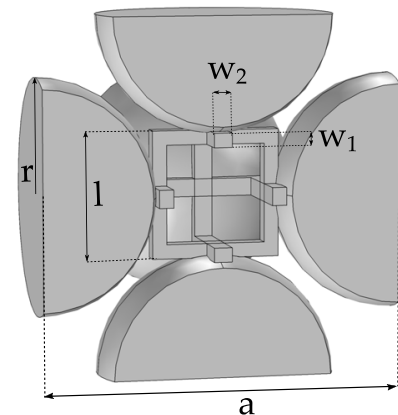

(b)
FIG. 1. Analysed periodic structure: (a) prototype in Nylon PA12 ${ }^{19}$ of dimensions $0.150 \mathrm{~m} \times 0.150 \mathrm{~m} \times 0.150 \mathrm{~m}$ of the periodic structure composed of $3 \times 3 \times 3$ unit cells and (b) a unit cell cross-section to define the structural parameters. 
In the proposed structure of Fig. 1(a), the main longitudinal wave propagation mechanism is characterized by spheres that move as rigid bodies and box-like connections that exploit the flexural stiffness of the beams [refer to Fig. 2(b)]. A one-dimensional mono-atomic spring mass chain approximation is introduced to model such a wave propagation phenomenon [refer to Fig. 2(a)]. The parameters $m$ and $k$ of such a chain are defined as the mass of the sphere, $m=4 / 3 \rho \pi r^{3}$, and the longitudinal stiffness of the box-like connection, $k=\alpha \beta k_{s b}$, respectively. The coefficient $\alpha=1 / 2$ accounts for the combination of the stiffnesses associated with the two couples of beams (each couple is attached to one sphere), while $\beta=2$ accounts for the sum of the stiffnesses of the two orthogonal beams (the two beams attached to one sphere). The stiffness of the single beam is $k_{s b}=24 E I / l_{e l}^{3}$ [refer to Fig. 2(c)], where $I=1 / 12 w_{2} w_{1}^{3}, l_{e l}=l / 3-w_{1} / 2$. The rigid part is a representation of the beam's portion that is attached to the sphere. The non-dimensional frequency value of the low-passband limit calculated with this scheme is ${ }^{20}$ $f_{\text {nd }}=f a / v=2 /(2 \pi) \sqrt{k / m} a / v=0.06663$ (refer to the red dashed horizontal line in Fig. 3).

The numerical phononic band structure is calculated by means of the Solid Mechanics Module of COMSOL Multiphysics v5.3, and it is shown in Fig. 3: the first bandgap is characterized by a gap to mid-gap ratio of $159.2 \%$, with non-dimensional frequency limits equal to 0.06560 and 0.57815 . The analytical prediction of the first bandgap low limit differs only by $1.59 \%$ from the numerical one.

The first bandgap is the widest complete bandgap in terms of the gap to mid-gap ratio for three-dimensional elastic periodic structures. Moreover, the periodic structure exhibits an opening frequency of the bandgap that is half of the one reported in the previous paper, ${ }^{18}$ with the same material and dimensions. This is due to the more compliant connection between the spheres, which exploits the flexural stiffness of the connecting beams instead of the axial one.

The most important result of this paper is the conception of a periodic structure in which the bandgap limits are overstepped in the transmission spectrum, so that the bandgaps in Fig. 3 are basically merged, approaching the behaviour of a low-pass mechanical filter. Such a physical property is connected to the specific organization of the vibrating modes that are now thoroughly examined. The modes which define each bandgap limit in the phononic band structure of Fig. 3 are reported in Fig. 4. It must be noticed that the mechanical

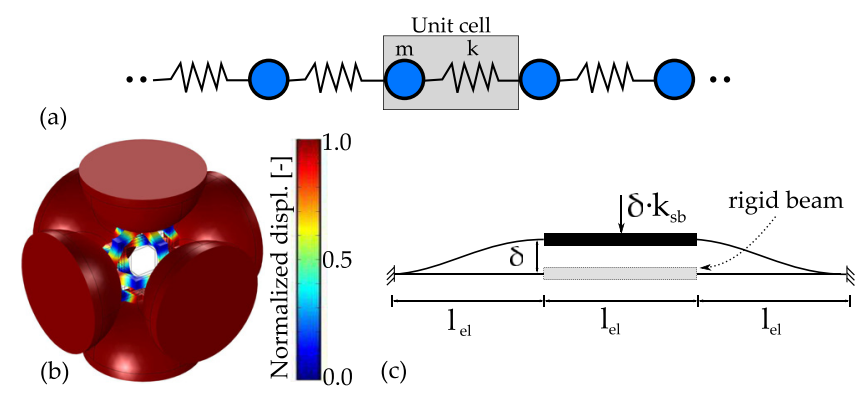

FIG. 2. Spring mass chain model: (a) mono-atomic spring-mass chain, (b) assumed mode shape (normalized with respect to the maximum displacement) for longitudinal wave propagation in the periodic structure, and (c) its simplified structural model.

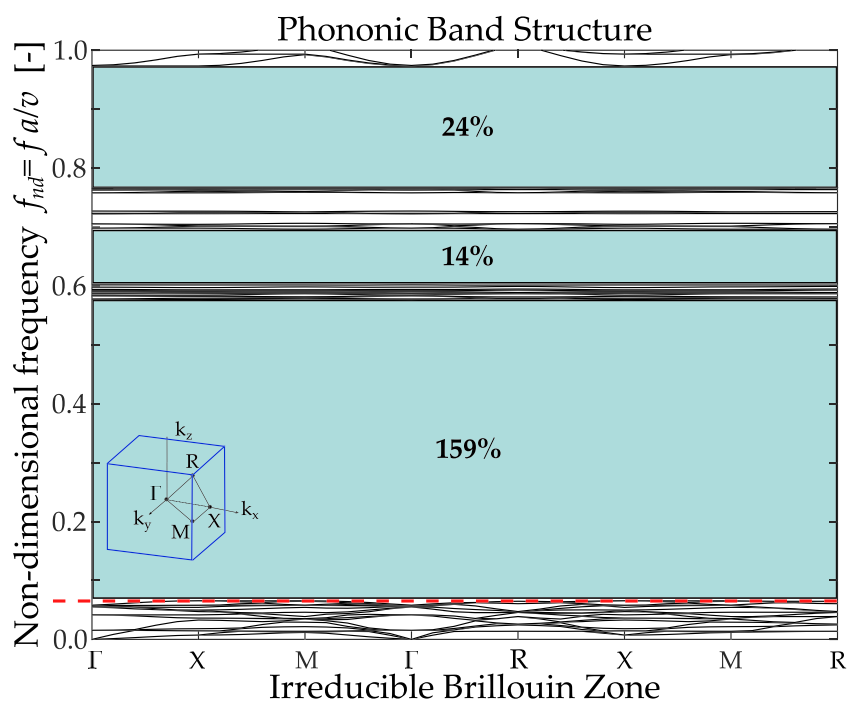

FIG. 3. Phononic band structure of the periodic structure: $f$ is the dimensional frequency and $v=\sqrt{E / \rho}$ sound velocity in the medium. The horizontal red dashed line is the analytical approximation of the bandgap opening frequency obtained with the spring-mass chain model shown in Fig. 2(a). The considered Irreducible Brillouin Zone (IBZ) is provided.

features of the modes in the limit bands (i.e., deformation mechanisms in terms of modal masses and modal stiffnesses) do not change significantly from one symmetry point to the other, the only differences being the propagation direction and the relative phase between periodically repeated points.

The opening mode of the first bandgap involves the whole unit cell, and it is therefore labelled as "global": the spheres can be seen as lumped masses with rigid motion, while the connecting beams play the role of elastic springs, undergoing flexural deformation. This mode, which is perfectly interpreted by the simple model in Fig. 2, occurs at low frequency mainly due to the significant modal mass. The closing mode of the first bandgap (refer to Fig. 4) is characterized by a local deformation of the box-like connection, while the spheres are almost unaffected. The modal mass is significantly lower than the one of the opening mode, while the modal stiffnesses are comparable, relying mainly on the flexural deformation of the beam connections. As a consequence, this mode is characterized by high frequency, and it

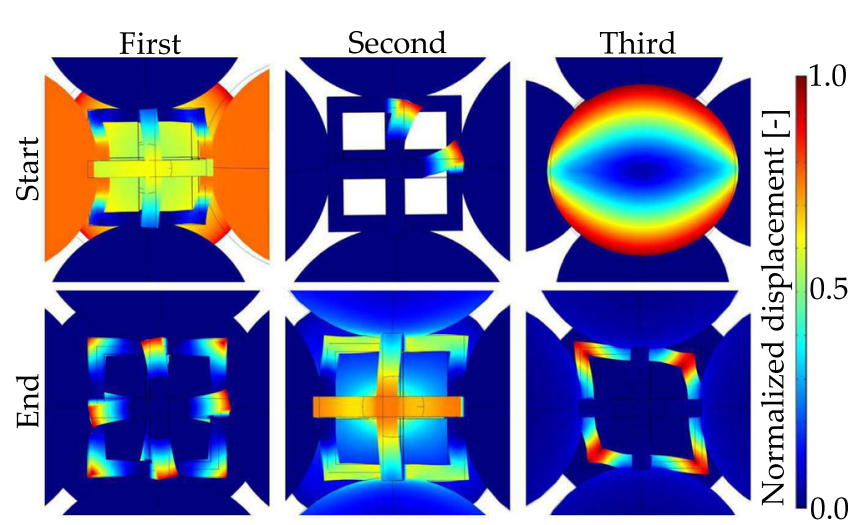

FIG. 4. Elastic mode shapes (normalized with respect to the maximum displacement) at lower and upper bounds of the bandgaps reported in Fig. 3. The symmetry points are not specified since the modes of each bandgap limit present the same mechanical characteristics. To improve the understanding of the mode shapes, the frontal semi-sphere is omitted where necessary. 
is called "local" since it does not involve the entire unit cell. Being the modal masses associated with boundaries of the first bandgap significantly different, an ultra-wide complete three-dimensional bandgap arises, as in the case of the diatomic spring-mass chain described in the literature. ${ }^{21}$ Overall, the infinite periodic structure is characterized by a set of global modes at low frequencies, ranging from the rigid body motions to all the modes involving the spheres as modal masses, and several bands of local modes at higher frequencies, each one implying the deformations of single components of the unit cell. More specifically, the second passband, as already commented, presents local modes that involve the connecting beams, while the third passband mainly presents modes with local deformations of the spheres (refer to Fig. 4). It must be stressed that the mode at the upper limit of the first bandgap and all the modes in the second passband are characterized by the wave propagation that does not involve the motion of the spheres (refer to Fig. 4), although complying with the Floquet-Bloch boundary conditions: for the periodic material architecture, this leads to the conclusion that few repetitions of the unit cell are sufficient to obtain a drastic reduction of the wave propagation also in this frequency range.

To confirm such a behaviour, a prototype of the considered structure [see Fig. 1(a)] made of $3 \times 3 \times 3$ unit cells and unit cell length $a=0.05 \mathrm{~m}$ is manufactured using Selective Laser Sintering (SLS) with Nylon PA12. ${ }^{19}$ This finite structure is both numerically analysed and experimentally tested to assess the transmission spectrum with reference to the $\Gamma$-X direction of the Irreducible Brillouin Zone (IBZ): ${ }^{18}$ the input and output reference areas are shown in Figs. 5(b) and 5(c), respectively.

The numerical transmission spectrum for the periodic structure is calculated by means of both the Solid Mechanics Module of COMSOL Multiphysics v5.3 and ABAQUS CAE v6.13 to double-check the results, by applying a harmonic force, and considering first a purely linear elastic behaviour

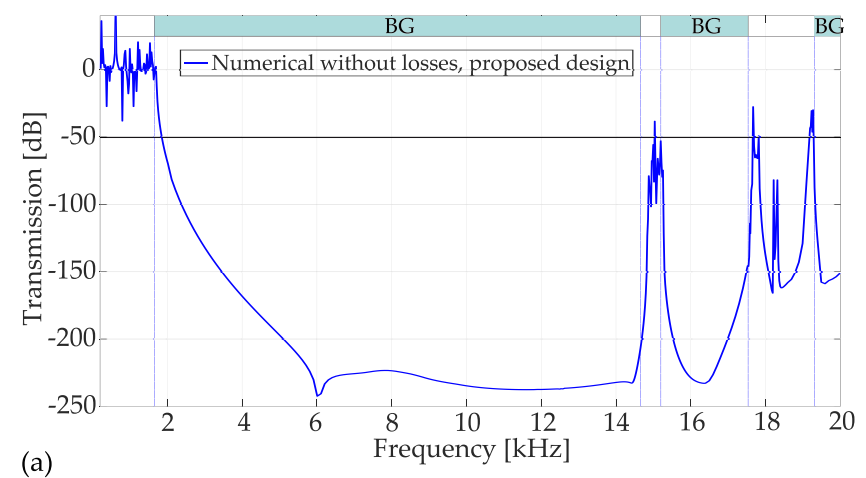

(a)

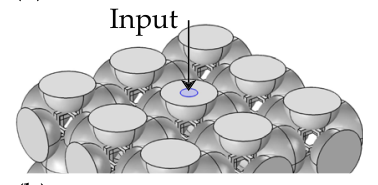

(b)

(c)

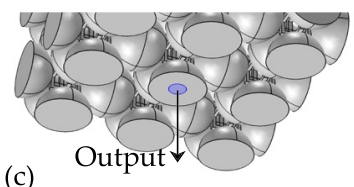

FIG. 5. Transmission analysis: (a) Numerical linear elastic transmission spectrum of the proposed design without losses (blue solid line) in the $\Gamma-\mathrm{X}$ IBZ path. The frequency limits of the (linear elastic) bandgaps reported in Fig. 3 are highlighted by vertical blue dashed lines. (b) Numerical and experimental reference area for the input signal. (c) Reference area for the output signal. of the Nylon to demonstrate the bandgaps merging without the additional effect of material loss. The numerical linear elastic transmission spectrum without losses is reported in Fig. 5(a) as the blue solid line, while the boundary frequency of the bandgaps is highlighted with vertical blue dashed lines: the two are in good agreement in terms of wave attenuation frequency bands. Although the prototype is made of just $3 \times 3 \times 3$ unit cells, the transmission spectrum presents a level of attenuation up to 11 orders of magnitude between the input and output accelerations in the first two bandgap regions. It must be pointed out that the numerical output signal is far from the machine precision in both the Finite Element solvers. Therefore, the attenuation up to $230 \mathrm{~dB}$ is considered reliable. It can be seen that the numerical transmission spectrum without losses presents a clear attenuation (of around $75 \mathrm{~dB}$ with $40 \mathrm{~dB}$ spikes) in the second passband: this confirms that the local modes that characterize this passband cannot be excited properly by the input force. The same concept applies to the third passband: to emphasize this effect, a bold line is drawn in correspondence of $50 \mathrm{~dB}$ of attenuation. This result vouches that it is possible to overstep the top limits of the bandgaps, and the related passbands, in three-dimensional elastic periodic structures, at least along the $\Gamma$-X IBZ path.

Experimental tests are conducted on the Nylon prototype: the excitation is applied in the orthogonal direction with respect to the input surface using an inertial actuator, a VibeTribe-Mamba with $20 \mathrm{~W}$ power and a frequency range from $40 \mathrm{~Hz}$ to $20 \mathrm{kHz}$. Two PCB Piezotronics 353B15 accelerometers, with a sensitivity of $10 \mathrm{mV} / \mathrm{g}$ and a resonant frequency of $70 \mathrm{kHz}$, are glued at the centers of the top [input, refer to Fig. 5(b)] and bottom [output, refer to Fig. 5(c)] faces of the prototype. The data acquisition chain is completed by an 8-channel PCB Piezotronics $483 \mathrm{C} 05 \mathrm{ICP}^{\circledR}$ Sensor Signal Conditioner and a NI 9205 module, with 16bit resolution. Tests are performed with a slow sweep sine input from $0.2 \mathrm{kHz}$ to $20.0 \mathrm{kHz}$, both sweep up and sweep down. A user routine in Matlab performs the post processing. It must be noticed that the maximum attenuation that can be measured with the current experimental setup is of $75 \mathrm{~dB}$ (i.e., 3.75 orders of magnitude).

The experimental transmission spectrum (thick black solid line in Fig. 6) clearly shows that the finite structure

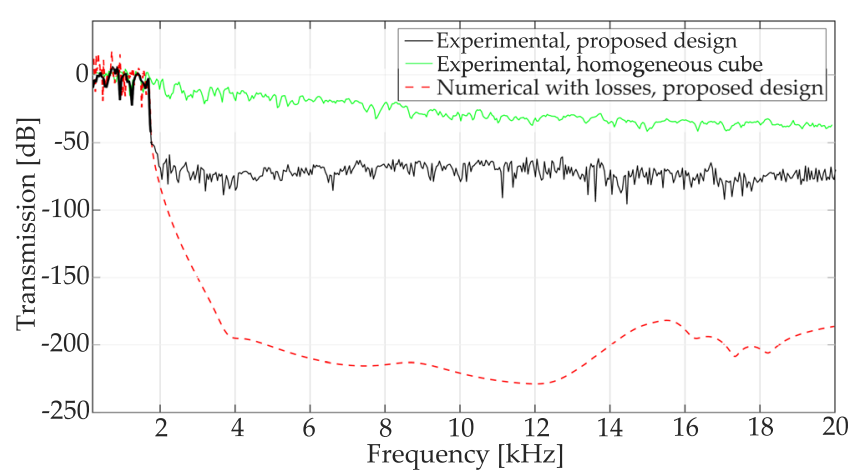

FIG. 6. Transmission analysis: the experimental transmission spectrum of the proposed design (thick black solid line) and of the homogeneous cube (thin green solid line) and numerical transmission spectrum of the proposed design considering losses (red dashed line). All the transmission spectra are along the $\Gamma-\mathrm{X}$ IBZ path. 
behaves like a mechanical low-pass filter in the audible frequency range: after the bandgap opening, the output signal remains around 3.75 orders of magnitude lower than the input until $20 \mathrm{kHz}$, with only 3 cell periodicity.

To investigate damping and other bulk material properties of the Nylon PA12, a second prototype, a homogeneous solid cube of the same dimensions, is manufactured with SLS: its transmission loss quantifies the bulk characteristics of the material (e.g., damping as a function of the excitation frequency), independently from the geometry of the structure. This comparison is sound since the smallest dimension in the periodic structure is sufficiently larger than the pixel precision of the SLS technique procedure, which are $2.0 \mathrm{~mm}$ and $0.1 \mathrm{~mm}$, respectively. The solid cube presents a smoothly decreasing transmission spectrum in the $\mathrm{dB}$ scale versus frequency (refer to the thin green solid line of Fig. 6): this indicates that the transmission loss increases as frequency increases. This characteristic is used to define a frequencyproportional loss factor for the Nylon PA12. A complex stiffness modulus is introduced, namely, $\tilde{E}=E(1+i \eta \omega)$, where $i$ is the imaginary unit, $\omega$ is the circular frequency, and the parameter $\eta$ has been obtained through the analysis of the transmission loss on the homogeneous cube. The identified value is $\eta=1.5 \mu \mathrm{s}$.

The numerical transmission spectrum considering losses is reported as the red dashed line in Fig. 6, showing that the amplitudes of the frequency response related to the second passband and above are beyond the experimental threshold of $75 \mathrm{~dB}$ and therefore cannot be captured by the experiment.

In this work, the merging of subsequent bandgaps in a three-dimensional, single material, elastic periodic structure is presented, approaching the behaviour of a low-pass mechanical filter for elastic waves in the audible frequency range. The presented design is endowed with the widest bandgap in terms of the gap to mid-gap ratio for 3D elastic periodic structures. The strategy to obtain such properties is described, relying on the modal analysis of the unit cell and on structural separation of global and local mode bands. This design leads to a generation of periodic structures to be used in robust and reliable wave attenuation over a wide frequency band.

The authors would like to thank M\&SS and LPMS labs of Politecnico di Milano for the precious support during the experimental tests and ZARE srl for the accurate fabrication of the prototypes. A.C. and L.D'A. acknowledge the contribution of MIUR: Project PRIN15-2015LYYXA8 Multiscale mechanical models for the design and optimization of microstructured smart materials and metamaterials. L.D'A. acknowledges Professor G. Della Valle of Politecnico di Milano for the fruitful discussion.

${ }^{1}$ E. Yablonovitch, T. J. Gmitter, and K. M. Leung, "Photonic band structure: The face-centered-cubic case employing nonspherical atoms," Phys. Rev. Lett. 67, 2295 (1991).

${ }^{2}$ J. Joannopoulos, S. Johnson, J. Winn, and R. Meade, Photonic Crystals: Molding the Flow of Light, 2nd ed. (Princeton University Press, 2011).

${ }^{3}$ P. Deymier, Acoustic Metamaterials and Phononic Crystals (SpringerVerlag, Berlin Heidelberg, 2013).

${ }^{4}$ V. Laude, Phononic Crystals: Artificial Crystals for Sonic, Acoustic, and Elastic Waves (De Gruyter, 2015).

${ }^{5} \mathrm{M}$. Maldovan, "Sound and heat revolutions in phononics," Nature 503, 209 (2013).

${ }^{6}$ L. Wang and B. Li, "Phononics gets hot," Phys. World 21, 27 (2008).

${ }^{7}$ F. Meseguer, M. Holgado, D. Caballero, N. Benaches, J. Sanchez-Dehesa, C. Lopez, and J. Llinares, "Rayleigh-wave attenuation by a semi-infinite two-dimensional elastic-band-gap crystal," Phys. Rev. B 59, 12169 (1999).

${ }^{8}$ A. Palermo, S. Krödel, A. Marzani, and C. Daraio, "Engineered metabarrier as shield from seismic surface waves," Sci. Rep. 6, 39356 (2016).

${ }^{9}$ A. Colombi, D. Colquitt, P. Roux, S. Guenneau, and R. Craster, “A seismic metamaterial: The resonant metawedge," Sci. Rep. 6, 27717 (2016).

${ }^{10}$ D. Colquitt, A. Colombi, R. V. Craster, P. Roux, and S. Guenneau, "Seismic metasurfaces: Sub-wavelength resonators and Rayleigh wave interaction,” J. Mech. Phys. Solids 99, 379 (2017).

${ }^{11}$ Y. Achaoui, B. Ungureanu, S. Enoch, S. Brûlè, and S. Guenneau, "Seismic waves damping with arrays of inertial resonators," Extreme Mech. Lett. 8, 30-37 (2016).

${ }^{12}$ Y. Achaoui, T. Antonakakis, S. Brûlè, R. V. Craster, S. Enoch, and S. Guenneau, "Clamped seismic metamaterials: Ultra-low frequency stop bands," New J. Phys. 19, 063022 (2017).

${ }^{13}$ Y. Pennec and B. Djafari-Rouhani, "Fundamental properties of phononic crystal," in Phononic Crystals: Fundamentals and Applications, edited by A. Khelif and A. Adibi (Springer, New York, NY, 2016), pp. 23-50.

${ }^{14}$ T. Delpero, S. Schoenwald, A. Zemp, and A. Bergamini, "Structural engineering of three-dimensional phononic crystals," J. Sound Vib. 363, 156-165 (2016).

${ }^{15}$ F. Lucklum and M. Vellekoop, "Band gap characterization of complex unit cell geometries for 3D phononic crystals," in 2016 IEEE International Ultrasonics Symposium (IUS) (2016), pp. 1-4.

${ }^{16} \mathrm{~S}$. Babaee, P. Wang, and K. Bertoldi, "Three-dimensional adaptive soft phononic crystals," J. Appl. Phys. 117, 244903 (2015).

${ }^{17}$ L. D'Alessandro, B. Bahr, L. Daniel, D. Weinstein, and R. Ardito, "Shape optimization of solid-air porous phononic crystal slabs with widest full 3D bandgap for in-plane acoustic waves," J. Comput. Phys. 344, 465-484 (2017).

${ }^{18}$ L. D'Alessandro, E. Belloni, R. Ardito, A. Corigliano, and F. Braghin, "Modeling and experimental verification of an ultra-wide bandgap in 3D solid-air phononic crystal," Appl. Phys. Lett. 109, 221907 (2016).

${ }^{19}$ SLS WhiteSinter ZARE srl, see http://www.zare.it/ for material characteristics.

${ }^{20}$ M. Hussein, M. Leamy, and M. Ruzzene, "Dynamics of phononic materials and structures: Historical origins, recent progress, and future outlook," Appl. Mech. Rev. 66, 040802 (2014).

${ }^{21}$ L. Brillouin, Wave Propagation in Periodic Structures: Electric Filters and Crystal Lattices (Dover Publications, Inc., New York, 1946). 